\title{
5G mm-Wave Link Range Estimation Based on Over-the-Air Measured System EVM Performance
}

\author{
Marko E. Leinonen, Nuutti Tervo, Markku Jokinen, Olli Kursu, and Aarno Pärssinen \\ University of Oulu, Center of Wireless Communications, Oulu, Finland \\ \{marko.e.leinonen, nuutti.tervo, markku.jokinen, olli.kursu, aarno.parssinen\}@oulu.fi
}

\begin{abstract}
Performance of 5G New Radio millimeter wave $(\mathrm{mmW})$ systems are expected to be tested over-the-air (OTA). In this paper, we use OTA measured system error vector magnitude (EVM) with varying automatic gain control, to characterize the achievable link range with 5G NR waveform. The platform used in the measurements is one of the first public $5 \mathrm{G} \mathrm{mmW}$ wireless backhaul Proof of Concept systems. We show that OTA system EVM measured in an Electro Magnetic Compatibility (EMC) chamber with varying AGC correlates well with line of sight outdoor measurements. Hence, the link range of the system can be approximated by attenuating OTA signal level either with gain controls of the radio units or with attenuation material between the radio units. The key advantage of link range estimation based on OTA measurements in the EMC chamber is better repeatability and protection from random radio interference which is present in real outdoor environment.
\end{abstract}

Keywords-Array EVM, Array receiver, OTA noise figure, Phased array, Wireless backhaul.

\section{INTRODUCTION}

The fifth generation $(5 \mathrm{G})$ communication system will offer ten-fold data rates compared with the current Long Term Evolution (LTE) system. The key enabler for enhanced data rates is that the $5 \mathrm{G}$ system will be deployed at millimeter wave $(\mathrm{mmW})$ bands where wider communication bandwidth can be used. The promise is to provide up to $20 \mathrm{Gbps}$ and $10 \mathrm{Gbps}$ peak data rates for downlink and uplink. The first trial $5 \mathrm{G}$ $\mathrm{mmW}$ networks were demonstrated in 2018 [1] and those are expected to be opened for commercial use during 2019.

High integration rate of the $\mathrm{mmW}$ transceivers and utilization of large antenna arrays challenges the traditional conducted testing that has been used in standardization of previous LTE, $3 \mathrm{G}$ and $2 \mathrm{G}$ systems. Different number of antennas from 32 [2] to 144 [3] or even 384 elements [4] have been reported in the literature. The cabling of antenna array for conducted testing would be very complex and the small physical dimensions of the array would make conducted testing unfeasible. For this reason, the $5 \mathrm{G}$ mmW NR (New Radio) standards [5],[6] utilize radiated measurements for characterizing the performance metrics used in standardization. The transition from conducted measurements to over-the-air (OTA) testing has drastic influence to radio system partitioning due to the fact that the measurements include also the effects of the antenna arrays. Utilizing $\mathrm{mmW}$ also simplifies OTA measurements due to the decreased far-field distance compared with the lower frequencies. Hence, even relatively large arrays can be measured inside a small Electro Magnetic Compatibility (EMC) chamber.
In LTE system, the error vector magnitude (EVM) is measured only at the highest transmission (TX) power level [7]. Moreover, the EVM of the receiver (RX) is not measured according to the standard since this parameter is validated via bit error rate (BER) or block error rate (BLER) measurements. However, relationship between BER/BLER and RX EVM is a non-linear function depending on the used signal modulation, coding, digital signal processing and RX signal level. The EVM gives a more consistent measure of RF performance than BER or BLER. The link range of the communication system depends on the received signal quality or the system EVM performance, which is a combination of the TX and RX EVMs [8]. Prestandard $5 \mathrm{G}$ prototype link range of $1.7 \mathrm{~km}$ at $28 \mathrm{GHz}$ has been reported in [9] and an inter-site distance of $5 \mathrm{G} \mathrm{mmW}$ system is expected to be $200 \mathrm{~m}$ [10]. This paper proposes that EMC laboratory measured system EVM performance with varying radiated input signal levels can be converted to expected link range.

\section{OVERVIEW OF 5G MMW PROOF-OF-CONCEPT SYSTEM}

The $5 \mathrm{G} \mathrm{mmW}$ wireless backhaul proof-of-concept (PoC) system used in the measurements includes a baseband signal processing unit for L2 and L3 processing, a digital radio unit for $\mathrm{L} 1$ processing and a radio unit. The radio unit includes two identical radio cards, each containing a 16 channel radio transceiver (TRX) which is updated version of TRX presented in [11]. The TRX architecture is a tree architecture with a power combining network at RF and a common path at RF and IF. The common path includes power control functions, frequency conversion and RF and IF filtering. The radio card is attached to an antenna module including 64 linearly polarized antenna elements shaped in $16 \times 4$ configuration. Hence, each TRX branch is driving a four-antenna subarray. The PoC radio unit supports beam steering of \pm 90 degrees out of which \pm 15 degrees is without significant grating lobes. The beam steering is implemented with phase shifters in each transceiver path. The control of the antenna beam is implemented with a software running on a dedicated processor board within the radio unit.

The main use case is that radio units are facing to each other at the boresight direction of the antenna array. A far-field threshold for an antenna can be calculated as follows [12]

$$
L \geq \frac{2 D^{2}}{\lambda},
$$

where $\mathrm{D}$ is a diameter of the minimum sphere enclosing the antenna, and $\lambda$ is a wavelength of the signal. A far-field threshold of the $5 \mathrm{G} \mathrm{mmW} \mathrm{PoC} \mathrm{antenna} \mathrm{array} \mathrm{is} 1.8 \mathrm{~m}$. 


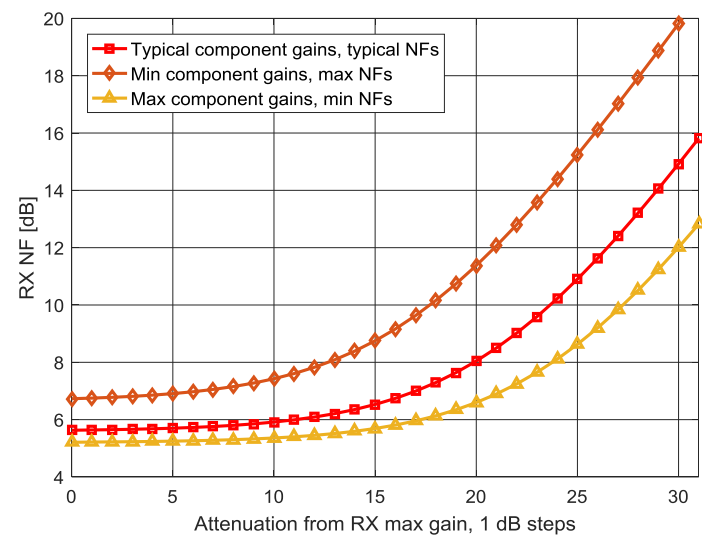

Fig. 1. RF system calculated of NF of array receiver with component variations with maximum coherence gain.

An automatic gain control (AGC) is implemented separately for TX and RX. The AGC is implemented with digitally controlled 5-bit step attenuators with $31 \mathrm{~dB}$ attenuation range at $\mathrm{mmW}$ frequency. The $\mathrm{AGC}$ controls both combined TX and RX signal levels of the whole array. Noise figure (NF) of the array receiver increases when the gain of the receiver is reduced. The RF system calculated NFs of the receiver array, including component variations within the specifications and routing losses, are shown in Fig. 1. The noise figure of the receiver path prior the first power combination is $4.9 \mathrm{~dB}$ with typical component values. Components including passive ones after the first power combining have a minor contribution to the total array NF. The gain of the receiver can be lowered $15 \mathrm{~dB}$ from the maximum RX gain without significant impact to NF.

Performance of the $5 \mathrm{G} \mathrm{mmW} \mathrm{PoC} \mathrm{has} \mathrm{been} \mathrm{validated} \mathrm{with}$ laboratory and outdoor measurements. Photographs of the complete $5 \mathrm{G}$ PoC setups in laboratory and outdoors are shown in Fig. 2a and Fig. 2b, respectively. A rack of measurement equipment used for EVM measurements is shown in Fig $2 b$.

\section{SYSTEM EVM MEASUREMENTS}

\section{A. System EVM Performance of Measurement System}

Radio link distance depends on the signal quality over the link and the main contributors are: radiated power from the TX, signal attenuation between TX and RX units, the NF of the RX and EVMs of TX and RX. The noise figure of the antenna array has been studied in [13] and OTA testing of it in [14].

The system EVM is combination of TX and RX EVMs [8] and additional contribution of measurement system EVM

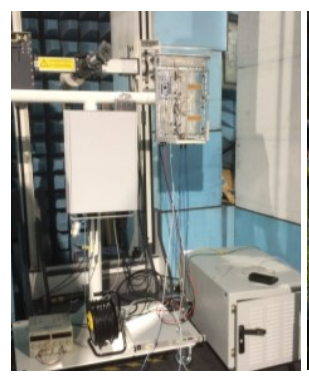

(a)

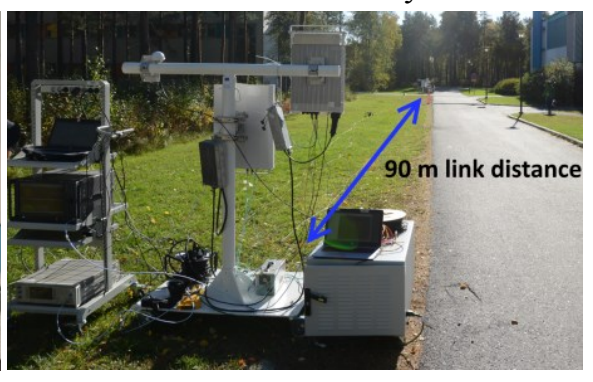

(b)
Fig 2. $5 \mathrm{G} \mathrm{mmW} \mathrm{PoC} \mathrm{testing} \mathrm{(a)} \mathrm{in} \mathrm{EMC} \mathrm{chamber} \mathrm{and} \mathrm{(b)} \mathrm{at} \mathrm{outdoor.}$

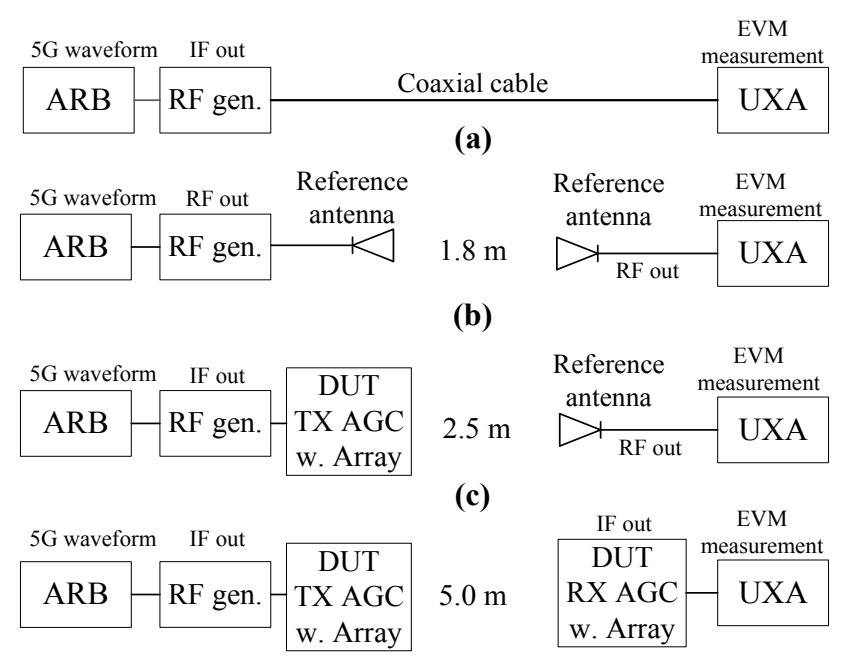

(d)

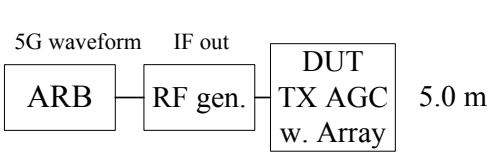

(e)

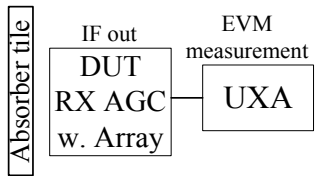

Fig. 3. EVM measurement block diagrams (a) basic conducted EVM (b) basic OTA, (c) TX EVM only, (d) system EVM in EMC chamber and (e) system EVM in EMC chamber with absorber tile.

should be included into analysis when measurement results are analysed. If EVM contributions are uncorrelated to each other, which is a valid assumption in most of the cases, then EVMs can be combined as follows

$$
\sqrt{E V M_{\text {system }}\left(P_{R X}\right)=}
$$

where $E V M_{T X}, E V M_{R X}$ and $E V M_{R F E q u i p}$ are EVMs of TX, RX, and the measurement system, respectively, $P_{\mathrm{TX}}$ is transmission power level and $P_{\mathrm{RX}}$ is received signal level.

The system EVM measurement flow is described as follows: first, the conducted base EVM of the measurement system is verified and the additional OTA contribution is measured. Next, the standalone EVM of the TX is measured, which is followed by the RX EVM performance verification. The received signal level is varied by TX and RX AGCs in system EVM performance measurement. Finally, the applicability of AGC based signal level variation is verified with an absorbing tile measurement which has similar attenuation as AGC attenuation. The measurement flow from a) to e) with corresponding EVM measurement setups is shown in Fig. 3.

The 5G waveform following the 3GPP NR standard [7] is generated by using Keysight M8190A arbitrary waveform

Table 1. Measurement system EVM of 5G 64-QAM, BW $=100 \mathrm{MHz}$

\begin{tabular}{|c|c|c|}
\hline $\mathrm{BW}=100 \mathrm{MHz}$ & Conducted EVM [\%] & OTA EVM [\%] \\
\hline $\mathrm{f}_{0}=4.0 \mathrm{GHz}(\mathrm{IF})$ & 0.446 & - \\
\hline $\mathrm{f}_{0}=28.0 \mathrm{GHz}(\mathrm{RF})$ & 1.776 & 3.145 \\
\hline
\end{tabular}




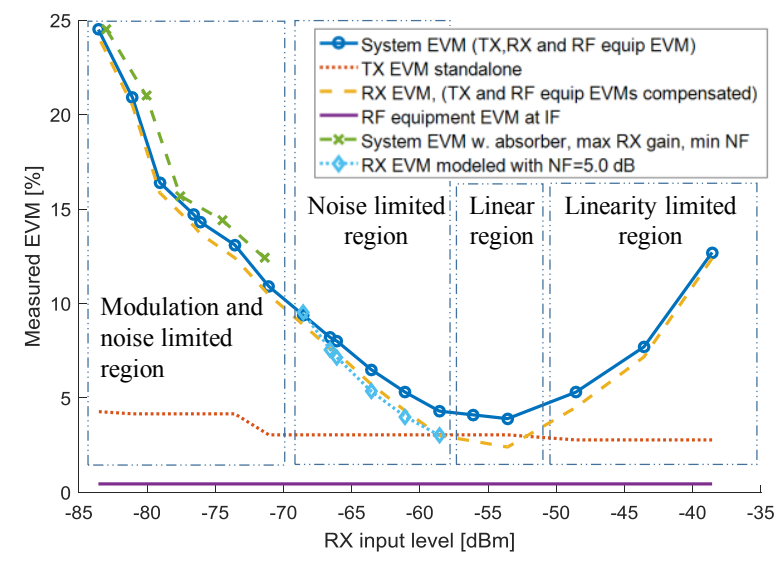

Fig. 4. System EVM OTA measurement of 5G PoC radio with 5G NR 64-QAM signal at $5 \mathrm{~m}$ distance in EMC laboratory.

generator (ARB). ARB is connected to RF signal generator ( $R F$ gen.) Keysight E8267D PSG by differential IQ signal for upconversion to RF and/or IF. The EVM is measured with a digital signal analyser Keysight N9040B (UXA). All EVM measurements have been done with $100 \mathrm{MHz}$ wide 16-QAM or 64-QAM Cyclic-Prefix Orthogonal Frequency Division Multiplexing (CP-OFDM) modulated signals. The $5 \mathrm{G}$ signal is fed into the $5 \mathrm{G}$ PoC radio at IF between 3 and $4 \mathrm{GHz}$ and the TX frequency is $28.0 \mathrm{GHz}$. The base EVM of the measurement equipment is measured as conducted by using the configuration shown in Fig 3a. The EVM of the measurement equipment is frequency dependent and hence different EVM contributions for different operational frequencies (IF or RF) are used. The base EVM of measurement equipment with OTA configuration is measured at $28.0 \mathrm{GHz}$ according to Fig. $3 \mathrm{~b}$ and the results are presented in Table 1. The OTA measurements have been done inside a $6 \mathrm{~m} \times 3 \mathrm{~m}$ EMC chamber in line of sight (LOS) condition. The EVM contribution due to OTA measurement over LOS channel can be calculated similar manner as in (2). The OTA EVM contribution is $2.60 \%$ due to e.g. channel estimation, equalisation and compensation filtering inside of the UXA and potential signal reflections inside of the EMC lab.

\section{B. System EVM Performance of $5 \mathrm{G} \mathrm{mmW}$ PoC}

The system EVM OTA measurements for the $5 \mathrm{G} \mathrm{mmW}$ PoC have been done with configurations presented in Fig. 3c, $3 \mathrm{~d}$ and $3 \mathrm{e}$. The TX EVM standalone performance was measured with a setup shown in Fig. 3c. The TX EVM varies from $2.78 \%$ to $4.27 \%$, minimum value reached at maximum TX power. The OTA EVM measurement has been done at 2.5 and $5 \mathrm{~m}$ distances, $5 \mathrm{~m}$ being the maximum practical distance within the EMC chamber. The measured system EVM in Fig. 3d and Fig. $3 \mathrm{e}$ cases includes the TX, the RX and the OTA EVM contributions. The RX EVM can be calculated from the measured system EVM results with (2) by subtracting the OTA and TX EVM contributions. The system EVM measurement was performed by sweeping the received signal level by varying the AGC settings of TX and RX. The effect of increasing NF at with high RX attenuation settings has not been compensated in system EVM analysis.

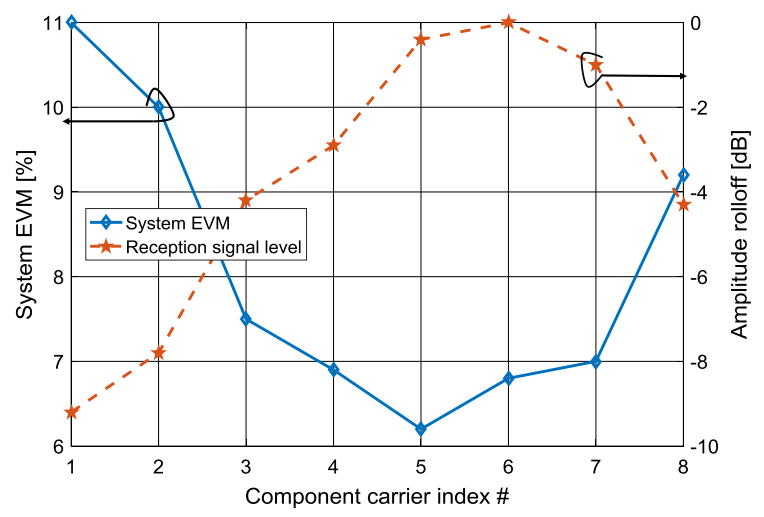

Fig. 5. System EVM of eight 64-QAM $100 \mathrm{MHz}$ component carrier signal on $28.0 \mathrm{GHz}$ center frequency at $-62.6 \mathrm{dBm}$ input level.

The measured system EVM curve with performance regions are shown in Fig. 4. The EVM performance is limited at high input powers by the linearity of the receiver and at low input power levels by the NF of the receiver. Between these regions there is an optimum EVM range of the system where the performance is dominated by phase noises and IQ-imbalances of TX and RX, and EVM contributions of digital signal processing in TX and RX. The best measured system EVM for the PoC system was $3.90 \%$.

The noise figure of the array has been calculated by curve fitting the thermal noise level with varying noise figure values as proposed in [14]. The EVM curve fitting was done for EVM values between $-58 \mathrm{dBm}$ and $-68 \mathrm{dBm}$, where the EVM curve follows a linear 1:1 slope on logarithm scale. The curve fitting was optimized with least squares method both on percent scale and on logarithmic scale and both curve fittings give $5.0 \mathrm{~dB}$ as a result for the array noise figure. At the lowest reception signal levels the higher order modulation of the signal flattens the EVM curve from 1:1 slope on logarithmic scale, when EVM is measured from constellation points without TX data comparison and the effect can be seen in the Fig. 4 and Fig. 7.

The AGC varied signal level test method was validated by system EVM measurements that were done with the maximum TX power with an absorber tile placed between TX and RX units as shown in Fig. 3e. Measured attenuation of the absorber tile was $32.8 \mathrm{~dB}$ at $28.0 \mathrm{GHz}$ frequency. The measured system EVM values with the absorber method match with TX and RX AGC varied system EVM measurement results between -70 $\mathrm{dBm}$ and $-81 \mathrm{dBm}$ as shown in Fig. 4. Based on these results, the AGC based method to modify the path loss between TX and $\mathrm{RX}$ by changing attenuator values is a valid method to virtually change the physical distance between the units.

The $5 \mathrm{G}$ PoC mmW radio supports signals up to $800 \mathrm{MHz}$ signal bandwidth with eight $100 \mathrm{MHz}$ component carriers (CC). The OTA system EVM measurement of eight CC with 64QAM was done and results are shown in Fig. 5. The IF frequency responses of the TX and RX create amplitude and EVM ripples to the measured results over eight $\mathrm{CCs}$. The $5 \mathrm{G}$ PoC radio supports four $100 \mathrm{MHz}$ 5G NR carriers similar EVM performance based on the results in Fig. 5. The four $\mathrm{CC}$ is the maximum configuration in current $5 \mathrm{G}$ NR standard [5]. 


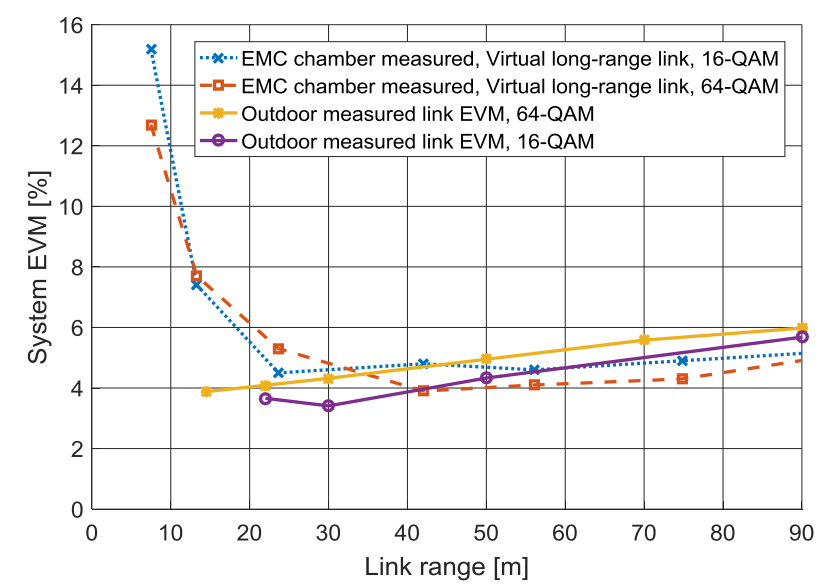

Fig. 6. EMC chamber and outdoor measurement comparison.

\section{IV.5G MMW PoC LINK RANGE EVALUATION BASED ON OTA SYSTEM EVM MEASUREMENTS}

The system EVM measurements done in the EMC chamber were validated with outdoor EVM measurements with the same TX power level. The outdoor measurements were done from 22 meters to 90 meters, which was limited by cable length and signal quality of the synchronization signal. The measurement results are shown in the Fig. 6. A good correlation of the system EVM values from EMC chamber and outdoor measurements can be seen. The maximum measured EVM difference between $90 \mathrm{~m}$ outdoor measurements and EMC chamber link model is $1.0 \%$. The link range estimations based on EMC chamber OTA measurement results are shown for both analysed 5G NR 16QAM and 64-QAM modulations at $100 \mathrm{MHz}$ bandwidth in Fig. 7. Maximum TX power (EIRP $45 \mathrm{dBm}$ ) is assumed for the link.

The EMC chamber measured system EVM link ranges are $332 \mathrm{~m}$ and $1095 \mathrm{~m}$ for $100 \mathrm{MHz}$ wide channel with 64-QAM modulation and 16-QAM modulation, respectively. The link ranges of four adjacent $\mathrm{CC}$ signals are scaled versions of the link range curves by $6 \mathrm{~dB}$ shown in Fig. 7. The estimated link ranges with four CC configuration are $166 \mathrm{~m}$ and $548 \mathrm{~m}$ with 64-QAM and 16-QAM modulation, respectively.

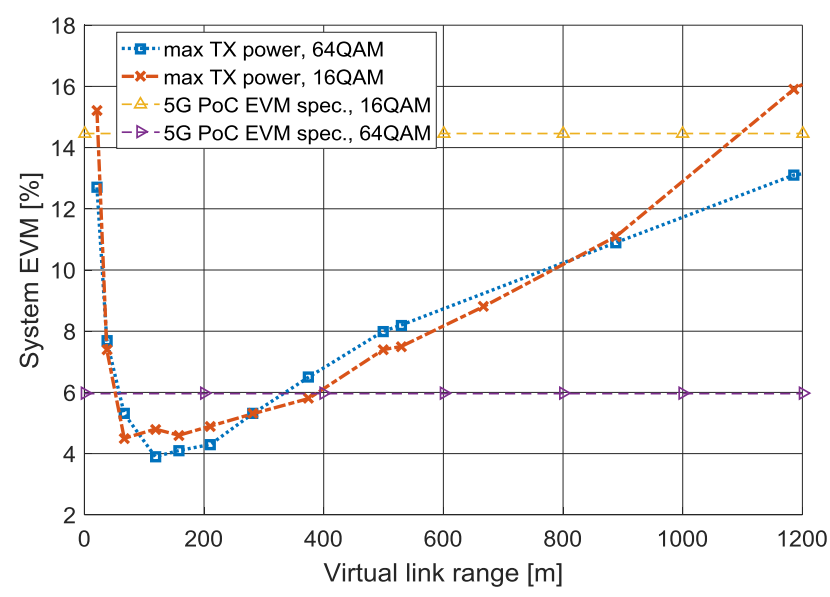

Fig. 7. Estimated link ranges of 5G NR 16-QAM and 64-QAM signals based on EMC laboratory EVM measurements.

\section{CONCLUSION}

The range of the wireless link is specified by system EVM which is a combination of TX and RX EVMs. Maximum allowed system EVM depends on used modulation, coding and signal processing algorithms. It was demonstrated that TX and RX AGCs can be used to virtually extend the distance between TX and RX by increasing signal attenuation between TX and RX. A good agreement of system EVM results measured in EMC chamber and 90 meter outdoor testing has been reported. The OTA system EVM performance measurement in EMC chamber enables reliable link range estimations to be performed indoors without time consuming outdoor measurements.

The estimated link ranges for the $5 \mathrm{G} \mathrm{PoC}$ system are $332 \mathrm{~m}$ with 64-QAM and $1095 \mathrm{~m}$ with 16-QAM modulations at 28 $\mathrm{GHz}$ frequency based on EMC OTA measurements. Four component carrier link ranges are estimated to be $166 \mathrm{~m}$ with 64-QAM and $548 \mathrm{~m}$ with 16-QAM modulations.

\section{ACKNOWLEDGMENTS}

The research leading to these results has received funding from the European Union H2020 5GPPP under grant n. 723247 and supported by the Institute for Information \& communications Technology Promotion (IITP) grant funded by the Korea government (MSIP) (No.B0115-16-0001, 5GCHAMPION). Also, this work was supported by the Academy of Finland 6Genesis Flagship (grant no. 318927).

\section{REFERENCES}

[1] "AT\&T Completes World's First mmWave Mobile 5G Browsing Session with Standards-Based Mobile Device," AT\&T Inc., Oct. 2018

[2] B. Sadhu et al., "A 28-GHz 32-Element TRX Phased-Array IC With Concurrent Dual-Polarized Operation and Orthogonal Phase and Gain Control for 5G Communications," in IEEE J. of Solid-State Circuits, vol. 52, no. 12, pp. 3373-3391, Dec. 2017.

[3] T. Sowlat et. al., "A 60GHz 144-Element Phased-Array Transceiver with $51 \mathrm{dBm}$ Maximum EIRP and $\pm 60^{\circ}$ Beam Steering for Backhaul Application," in ISSCC2018, San Francisco, CA, pp. 66-68

[4] S. Shahramian et. al. "A Fully Integrated Scalable W-Band PhasedArray Module with Integrated Antennas, Self-Alignment and Self-Test," in ISSCC2018, 11. - 15. Feb. 2018, San Francisco, CA, pp. 74 - 76

[5] Base Station (BS) radio transmission and reception (Release 15) 3GPP TS 38.104 V15.2.0, Jun. 2018.

[6] User Equipment (UE) radio transmission and reception, Part 2: Range 2 Standalone (Release 15) 3GPP TS 38.101 V15.2.0, Jun. 2018.

[7] Base Station (BS) radio transmission and reception (Release 15) 3GPP TS 36.104 V15.1.0, Dec. 2017.

[8] T. Tuovinen, N. Tervo and A. Pärssinen, "Analysing 5G RF System Performance and Relation to Link Budget for Directive MIMO," IEEE Trans. Antennas Propag., vol. 65, no. 12, Dec. 2017, pp. 6636 - 6645.

[9] W. Roh et al.,"Millimeter-wave beamforming as an enabling technology for $5 \mathrm{G}$ cellular communications: theoretical feasibility and prototype results," in IEEE Comm. Mag., vol. 52, no. 2, pp. 106-113, Feb. 2014

[10] "Mobilizing 5G NR Millimeter Wave: Network Coverage Simulation Studies for Global Cities," Qualcomm Inc., white paper, Oct. 2017

[11] O. Kursu et al., "Design and measurement of a $5 \mathrm{G} \mathrm{mmW}$ mobile backhaul transceiver at $28 \mathrm{GHz}$, EURASIP J. Wirel. Comm., Vol. 2018, No. 1, Aug. 2018.

[12] C. A. Balanis, Modern Antenna Handbook. John Wiley \& Sons Inc., USA, 2008.

[13] J. J. Lee, "G/T and Noise Figure of Active Antennas," IEEE T. Antennas Propag., vol. 41, no. 2, Feb. 1993, pp. 241-244.

[14] M. E. Leinonen, G. Destino, O. Kursu, M. Sonkki and A. Pärssinen, " $28 \mathrm{GHz}$ Wireless Backhaul Transceiver Characterization and Radio Link Budget," ETRI J., Feb. 2018, 40: 89-100. 